Originales

\title{
Adenocarcinoma colorrectal: factores pronósticos de supervivencia en el tratamiento adyuvante con 5-Fluorouracilo-Levamisol (5FU-Lev)
}

\author{
C. Bosch Roig*, M. M. Morales Suárez-Varela**, C. Molins Palau*, D. Almenar Cubells*, S. Olmos Antón*,
} J. Muñoz Langa*, M. Tallón Guerola*

\section{Resumen}

- Propósito: Valoración de la supervivencia global (SG) y libre de enfermedad (SLE) a 5 años y estudio de los factores pronósticos de los pacientes operados, con finalidad curativa, de adenocarcinoma colorrectal estadios II y III, que recibieron tratamiento adyuvante con 5FU-LEV.

- Material y métodos: ciento veintiséis pacientes tratados con esquema 5FU-LEV durante 12 meses, entre septiembre de 1990 y noviembre de 1997.

- Resultados: La SG a los 5 años es de $68.3 \%$ y la SLE de 54\%. El número de ganglios afectos por el tumor y la afectación vásculo-linfática y perineural, han resultado factores pronósticos significativos tanto en la SLE como en SG a los 5 años. La existencia de obstrucción intestinal tan sólo resulta factor pronóstico significativo en la SLE a los 5 años.

- Conclusiones: los resultados que presentamos son semejantes a otras grandes series conocidas, tanto en supervivencia global y libre de enfermedad como en el estudio univariante de los factores pronósticos. La afectación ganglionar continúa presentando significación pronóstica con el análisis multivariante.

Palabras clave:

Cáncer colorrectal. Quimioterapia adyuvante. Factores pronósticos.

Oncología, 2004; 27 (8):490-498 


\section{Summary}

- Purpose: To assess the overall survival and the 5-year disease-free survival, and to investigate the prognostic factors of patients with stages II and III colorectal adenocarcinoma subjected to healing-intention surgery and adjuvant chemotherapy with 5-fluorouracil-levamisole (5-FU-LEV).

- Material and methods: A cohort of 126 patients treated with 5-FU-LEV during 12 months were studied between September 1990 and November 1997.

- Results: The number of infiltrated lymph nodes, and the vascular-lymphatic and perineural invasion were found to be significant prognostic factors in the mean overall survival and disease-free survival after 5 years. Bowel obstruction showed prognostic significance only in relation to the 5 -year disease-free survival.

- Conclusions: The obtained results are similar to those reported in other large series published in the literature related with overall survival, disease-free survival and prognostic factors. Infiltrated lymph nodes remain as a significant prognostic factor in multivariate analysis.

Key words: Colon cancer. Rectal cancer. Adjuvant chemotherapy. Prognosis study.

\section{Introducción}

El carcinoma colorrectal es una enfermedad frecuente en los países occidentales. El $77 \%$ de los casos se diagnosticaron en estadios en los que es posible practicar una resección radical del tumor, no obstante, sin tratamiento adyuvante, hasta un $50 \%$ de estos pacientes recidivarán y morirán por causa del tumor ${ }^{1,2}$. El principal factor pronóstico es el estadio tumoral, según la clasificación TNM de la International Union Againts Cancer (UICC) ${ }^{3}$.

Tras varios intentos, en 1988, Buyse et al ${ }^{4}$ publicaron el primer meta-análisis en el que se demostró un escaso beneficio del tratamiento adyuvante con $5 \mathrm{FU}$ en cáncer de colon.

Posteriormente, tanto el estudio del North Central Center Treatment Group (1998) por Moertel et al del Intergroup (1990) ${ }^{6}$, evidenciaron una mejoría tanto del intervalo libre de enfermedad (ILE) como de la SG en pacientes con carcinoma de colon en estadio III tratados con 5FU Levamisol durante un año, respecto a los pacientes que no se trataron o recibieron sólo Levamisol.

En los tumores de recto la radioterapia adyuvante disminuye las recidivas pélvicas, aunque no aumente la supervivencia global. Los estudios de los grupos GITSG $^{2}$ y Mayo/NCCTG, demostraron que la combinación quimio-radioterapia con 5-FU era superior a la radioterapia postoperatoria y a la cirugía sola.

Ante estos resultados, la Conferencia de Consenso del National Institute of Health de USA (1990$1991)^{6,7,8}$, recomendó el tratamiento adyuvante con 5FU-Levamisol en los adenocarcinomas de colon en estadio III, y † con 5FU y radioterapia pélvica en los tumores de recto en estadios II y III.

El objetivo de nuestro trabajo es la valoración de la supervivencia libre de enfermedad (SLE), la supervivencia global (SG) a los cinco años y el estudio de los factores pronósticos de pacientes intervenidos con finalidad radical de tumores colorrectales en estadios II y III, que recibieron tratamiento adyuvante con 5FU-Levamisol.

\section{Material y métodos}

Se realizó un estudio retrospectivo, en forma de cohorte histórica, de pacientes operados de adenocarcinoma de colon y recto con intención radical lescisión completa del tumor, con márgenes quirúrgicos adecuados y resección en bloque de ganglios regionales) tratados con quimioterapia adyuvante, entre septiembre de 1991 y noviembre de 1997, en el Hospital Universitario Dr. Peset de Valencia.

Se incluyeron tumores de colon en estadio II (T3NOMO con factores de mal pronóstico, obstrucción intestinal, adenocarcinomas indiferenciados, elevación del CEA preoperatorio y afectación vásculo-linfática y perineural y T4NOMO) y estadio III (cualquier T con afectación ganglionar, N1 y N2 MO) y los tumores localizados en recto en estadios II y III. Los pacientes debían tener un buen estado general (PS 0-2) y una adecuada reserva medular ( $\geq 4000$ leucocitos y 100.000 plaquetas en un hemograma reciente).

Los criterios de exclusión fueron: tumores colorrectales metastáticos y existencia previa de cualquier otro 


\section{Bosch Roig y cols.}

tumor (excepto un tumor superficial de piel que no fuera un melanoma o un carcinoma in situ de cérvix).

El tratamiento consistió en $450 \mathrm{mg} / \mathrm{m}^{2}$ de $5 \mathrm{FU}$ vía endovenosa durante cinco días y después de 4 semanas de descanso, se continuaba con una dosis semanal de 5FU a las dosis referidas, durante 1 año 6 . Además, los pacientes también recibían Levamisol $50 \mathrm{mg}$. vía oral cada 8 horas durante 3 días, repitiéndose cada 15 días durante un año.

Además del esquema quimioterápico anterior, los pacientes con tumores de recto recibieron radioterapia pélvica adyuvante (50cGy) con 3-4 campos opuestos y un fraccionamiento de 1.8 Gy por día, 5 días por semana durante 6 semanas.

Tras la finalización del tratamiento los pacientes acudieron a control cada 3 meses durante los dos primeros años y, después, cada 6 meses durante 5 años.

En cada visita de control se realizó anamnesis, exploración física y nivel de CEA (antígeno carcino-embrionario). Entre las exploraciones complementarias, se practicaron ecografías abdominales en tumores resecados en colon y TACs abdomino-pélvicos alternándose con radiografía de tórax, en los pacientes intervenidos de carcinomas de recto. La recto-colonoscopia de control se practicó cada 6 meses, durante los dos primeros años tras la cirugía y, posteriormente, una vez al año hasta el quinto año (a partir de 1997 su frecuencia disminuyó a cada 3 años si no existía ninguna sintomatología).

Se consideró recidiva la reaparición del tumor demostrada con métodos radiográficos (RMN y TAC abdomino-pélvico) o mediante exploración física. Se acompañó de estudios mediante PAAF o biopsia en casos de duda en las anteriores exploraciones. La mayoría de las recidivas se acompañaron de un aumento de los valores del CEA (si bien, la elevación del CEA únicamente, no fue suficiente para considerar una recidiva tumoral).

Se seleccionó información de 13 variables por cada paciente, estudiadas en frecuencia absoluta y relativa con un intervalo de confianza del 95\% (IC 95\%). La relación entre variables se realizó mediante pruebas tanto paramétricas como no paramétricas, pero siempre con una significación del 95\%. Los factores pronósticos analizados son: edad y sexo, existencia de obstrucción intestinal, CEA, hemoglobina y albúmina preoperatorios, localización del tumor en el intestino grueso, grado de diferenciación tumoral, afectación de las capas de la pared del colon y recto $(T)$, número de ganglios afectos por el tumor (N), invasión perineural y de vasos linfáticos intratumorales. También se analizaron la SG y SLE (calculada desde la fecha de la intervención quirúrgica) según los factores pronósticos, aplicando el cálculo del Riesgo Relativo (RR), el test estadístico de
Mantel-Haenzel9, 10, y las curvas de supervivencia de Kaplan-Meier ${ }^{11}$.

Por último, se realizó un análisis multivariante de Cox. Los criterios que se aplicaron en la inclusión de variables fueron, por una parte, las variables más relevantes con carácter pronóstico descritas en la literatura científica y, por otra, aquellas que en nuestra serie nos resultaron estadísticamente significativas en el análisis de Kaplan-Meier anteriormente realizado (concretamente estas fueron: niveles séricos de CEA y albúmina preoperatorias, afectación perineural y de vasos linfáticos por el tumor en la pieza quirúrgica, afectación ganglionar, grado de diferenciación tumoral y presencia de obstrucción intestinal). Este análisis multivariante nos permitió valorar aquella variable con un riesgo mas elevado de mortalidad y de recidiva tumoral. Se obtuvo la significación estadística con el Test de Wald (con un nivel de significación del 95\%).

\section{Resultados}

De los 138 pacientes seleccionados 12 fueron excluidos, 7 por presentar estadio metastásico, 3 por éxitus precoz (dos por IAM y uno por ACV) y 2 pacientes a los que se suspendió el tratamiento por toxicidad intolerable. El tamaño final de la muestra fue de 126 casos.

En la Tabla I se muestran las características clínicas y patológicas, así como su influencia sobre la SLE y la SG. Setenta y tres hombres y 53 mujeres. El 45\% (57 pacientes) eran mayores de 65 años. Su estado general fue bueno, 97 casos (77\%) con ECOG 0 y 19 (15\%) con ECOG 1.

La localización tumoral fue: colon ascendente y transverso en 27 pacientes $(21.5 \%)$, colon descendente y sigma en 61 pacientes $(48.5 \%)$, y 35 pacientes $(27.7 \%)$ presentaron el tumor en el recto. En 3 casos $(2.3 \%)$ se desconocía la localización dentro del intestino grueso.

Ochenta y siete pacientes fueron T3 (afectación de la serosa), y 34 fueron T4 (afectación de los órganos vecinos). En 4 casos se desconoce la afectación tumoral de la pared intestinal. Diecisiete pacientes presentaron obstrucción intestinal al diagnóstico.

El tumor fue G1 (bien diferenciado), G2 (moderadamente diferenciado) y G3 (indiferenciado) en 33, 74 y 7 casos respectivamente. No se conocía el grado de diferenciación tumoral en 12 pacientes.

En 50 de los casos (39.7\%) no existía afectación ganglionar (N0), 58 pacientes (46\%) tenían entre 1 y 4 ganglios afectos por tumor y en 11 casos (8.7\%) afectaba a más de 4 ganglios. En 7 pacientes no se informó del número de ganglios afectos.

De los 42 pacientes en estadio II de colon, todos 
TABLA I

Factores pronósticos seleccionados valorando el porcentaje de Supervivencia libre de enfermedad (SLE) y de Supervivencia global (SG) ambos a los 5 años

\begin{tabular}{|c|c|c|c|c|c|}
\hline Variables & n (126) & (\%) S.L.E.* & Valor P & (\%) S.G.* & Valor $\mathbf{P}$ \\
\hline \multicolumn{6}{|l|}{ Sexo } \\
\hline Hombres & 73 & 64 & & 72.0 & \\
\hline Mujeres & 53 & 61 & 0.253 & 62.2 & 0.445 \\
\hline \multicolumn{6}{|l|}{ Edad } \\
\hline $18-65$ & 69 & 62.7 & & 67.6 & \\
\hline$>65$ & 57 & 60.1 & 0.617 & 68.3 & 0.385 \\
\hline \multicolumn{6}{|l|}{ Estado general } \\
\hline 0 & 97 & 60.0 & & 64.8 & \\
\hline 1 & 19 & 56.1 & 0.832 & 71.0 & 0.094 \\
\hline \multicolumn{6}{|l|}{ Localización } \\
\hline Colon ascendente y transverso & 27 & 55.7 & & 58.4 & \\
\hline Colon descendente, sigma recto-sigma & 61 & 71.93 & & 72.58 & \\
\hline \multirow{2}{*}{\multicolumn{6}{|c|}{ Afectación intramural }} \\
\hline & & & & & \\
\hline T3 & 87 & 62.5 & & 67.9 & \\
\hline $\mathrm{T} 4$ & 34 & 56.4 & 0.578 & 66.4 & 0.329 \\
\hline \multicolumn{6}{|l|}{ Obstrucción intestinal } \\
\hline Sí & 17 & 61.2 & & 58.8 & \\
\hline No & 109 & 74.1 & 0.019 & 70.9 & 0.197 \\
\hline \multicolumn{6}{|l|}{ Grado diferenciación } \\
\hline G1 & 33 & 78.1 & & 71.8 & \\
\hline G2 & 74 & 57.2 & & 55.5 & \\
\hline G3 & 7 & 50.0 & 0.778 & 50.0 & 0.657 \\
\hline \multicolumn{6}{|l|}{ Afectación ganglionar } \\
\hline Ganglios negativos (NO) & 50 & 87.3 & & 87.9 & \\
\hline $1-4$ ganglios & 58 & 53.7 & & 58.9 & \\
\hline$>4$ ganglios & 11 & 45.4 & 0.003 & 45.4 & 0.001 \\
\hline \multicolumn{6}{|l|}{ Afectación vascular linfàtica } \\
\hline Sí & 27 & 51.9 & & 7.4 & \\
\hline No & 27 & 77.8 & 0.010 & 44.4 & 0.001 \\
\hline \multicolumn{6}{|l|}{ Afectación perineural } \\
\hline Sí & 12 & 50 & & 0 & \\
\hline No & 33 & 72.7 & 0.029 & 39.3 & 0.001 \\
\hline \multicolumn{6}{|l|}{ Hemoglobina preoperatoria } \\
\hline$\leq 12 \mathrm{~g} / \mathrm{dl}$ & 52 & 52.7 & & 56.2 & \\
\hline$>12 \mathrm{~g} / \mathrm{dl}$ & 59 & 64.1 & 0.520 & 71.1 & 0.584 \\
\hline \multicolumn{6}{|l|}{ Albúmina preoperatoria } \\
\hline$\leq 3.5 \mathrm{~g} / \mathrm{dl}$ & 16 & 70.3 & & 75.8 & \\
\hline$>3.5 \mathrm{~g} / \mathrm{dl}$ & 52 & 48.2 & 0.275 & 53.2 & 0.290 \\
\hline \multicolumn{6}{|l|}{ CEA preoperatorio } \\
\hline$\leq 5 \mathrm{ng}$ & 73 & 60.6 & & 64.4 & \\
\hline$>5 \mathrm{ng}$ & 25 & 53.8 & 0.810 & 58.3 & 0.096 \\
\hline
\end{tabular}

*Kaplan-Meier estimados a los 5 años de seguimiento.

presentaron algún factor de mal pronóstico: 14 casos presentaron obstrucción intestinal, 2 perforación de colon, 10 tuvieron un valor aumentado del CEA preoperatorio y 29 fueron T4.
En 27 casos hubo afectación vásculo-linfática, y en otros 27 casos no. En 12 adenocarcinoma colorrectales resecados hubo afectación perineural intratumoral, y en 33 pacientes no la hubo. En 81 casos se descono- 
cia este dato. El valor de la hemoglobina preoperatoria fue igual o menor de $12 \mathrm{gr} / \mathrm{dl}$ en 52 casos y mayor de este valor en 59 casos.

El nivel de la albúmina preoperatoria fue menor de $3.5 \mathrm{~g} / \mathrm{dl}$ en 16 pacientes y 52 por encima de este valor. El CEA preoperatorio estaba aumentado por encima del valor de referencia $(5 \mathrm{ng} / \mathrm{ml})$ en 25 pacientes y fue normal en 73 casos.

En los que presentaron clínica de obstrucción intestinal al diagnóstico, la SLE a los 5 años fue del $61.2 \%$, y del $74.1 \%$ si no tuvieron esta clínica $(p=0.019)$ (Fig. 1). Aunque la SG a los 5 años fue del $58.9 \%$ cuando los pacientes manifestaron un cuadro de obstrucción intestinal y del $70.9 \%$ cuando no lo presentaron, esta tendencia no consiguió una significación estadística $(p=0.197)$.

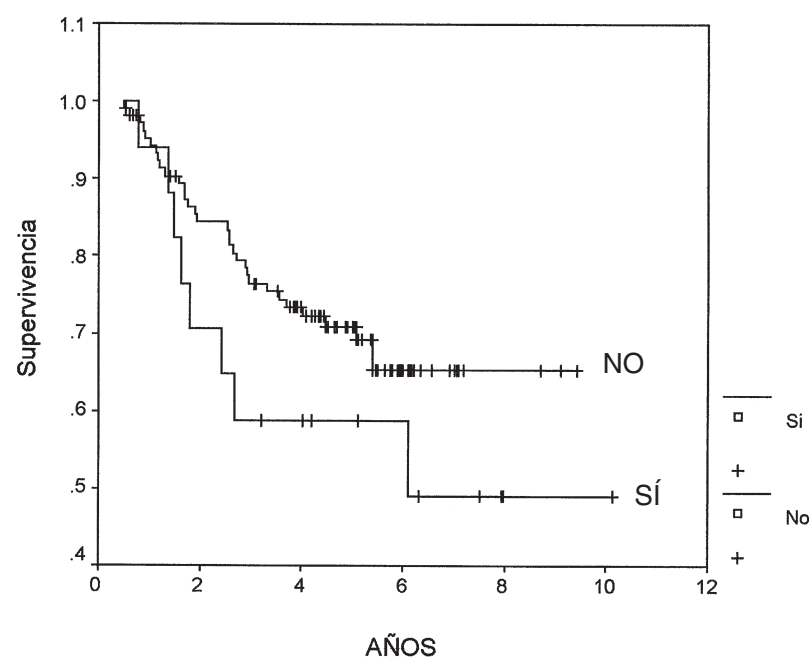

Fig. 1. Evolución del SLE de los enfermos tratados con 5FULev y presentaron clínica de obstrucción intestinal en el diagnóstico.

Entre las variables patológicas, el $78.1 \%$ de los pacientes con tumores $G 1$ estaban libres de enfermedad a los 5 años, respecto al $57.2 \%$ de G2 y del $50 \%$ en tumores $\mathrm{G} 3(\mathrm{p}=0.778)$. En cuanto a la supervivencia global, el $71.8 \%$ de los $\mathrm{G} 1$ están vivos a los 5 años respecto al $55.5 \%$ de los $\mathrm{G} 2$, y el $50 \%$ de los $\mathrm{G} 3$ ( $p=$ 0.657).

La afectación ganglionar se muestra como factor pronóstico significativo tanto en la SLE como en la SG a los 5 años. Los pacientes en estadio II (NO), están libres de enfermedad el $87.3 \%$ mientras que los que tenían entre 1 y 4 ganglios (N+1-4) afectos, la SLE a los 5 años fue de un $53 \%$. Con más de 4 ganglios afectos

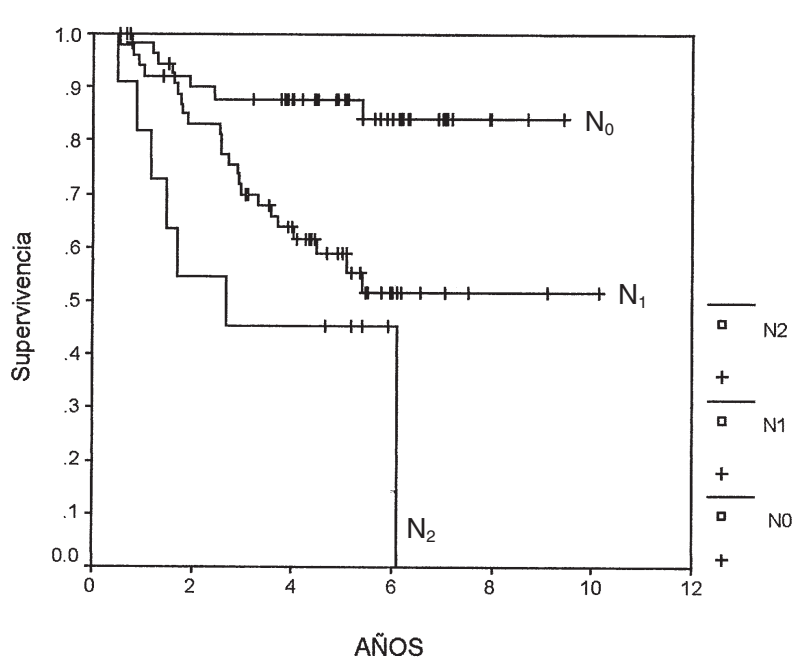

Fig. 2. Evolución del SLE de los pacientes de CCR tratados con 5FU-lev según la afectación ganglionar.

por tumor $\left(N_{+}>4\right)$, la SLE baja al $45.4 \%$ a los 5 años $(p=0.003)$ (Fig. 2).

La afectación ganglionar en los estratos $\mathrm{NO}, \mathrm{N}+1-4$ y $\mathrm{N}+>4$, presenta una $S G$ a los 5 años del $87.9 \%$, $58.9 \%$ y el $45.4 \%$ respectivamente, con significación estadística ( $p=0.001$ ) (Fig. 3).

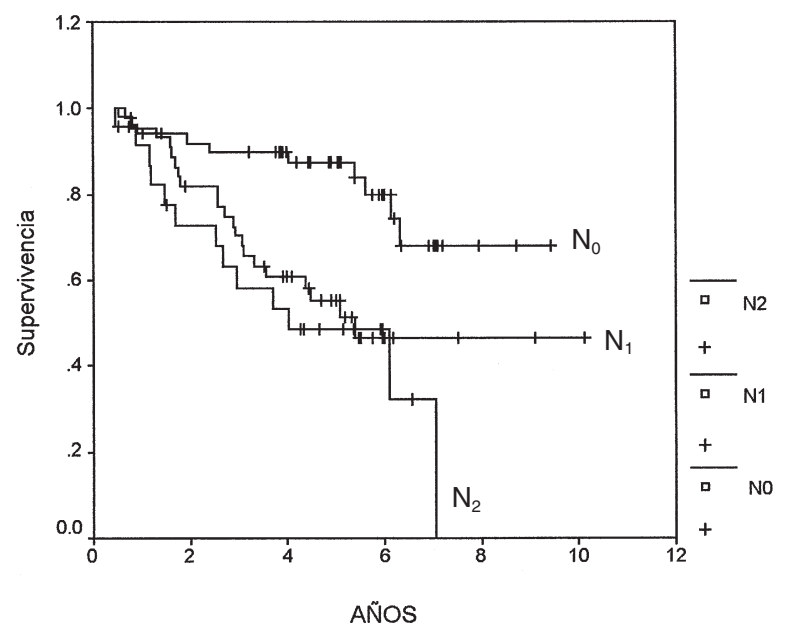

Fig. 3. Evolución de la SG de los pacientes de CCR tratados con 5FU-lev según la afectación ganglionar.

Los tumores con afectación vásculo-linfática, mostraron una SLE a los 5 años del $51.8 \%$ frente al $77.8 \%$ si no la presentaban ( $p=0.010)$. La SG fue del $7.4 \%$ a los 5 años en los pacientes que tenían afectación vásculo-linfática y del $44.4 \%$ en los que no la presentaban $(p=0.005)$ (Fig. 4). 


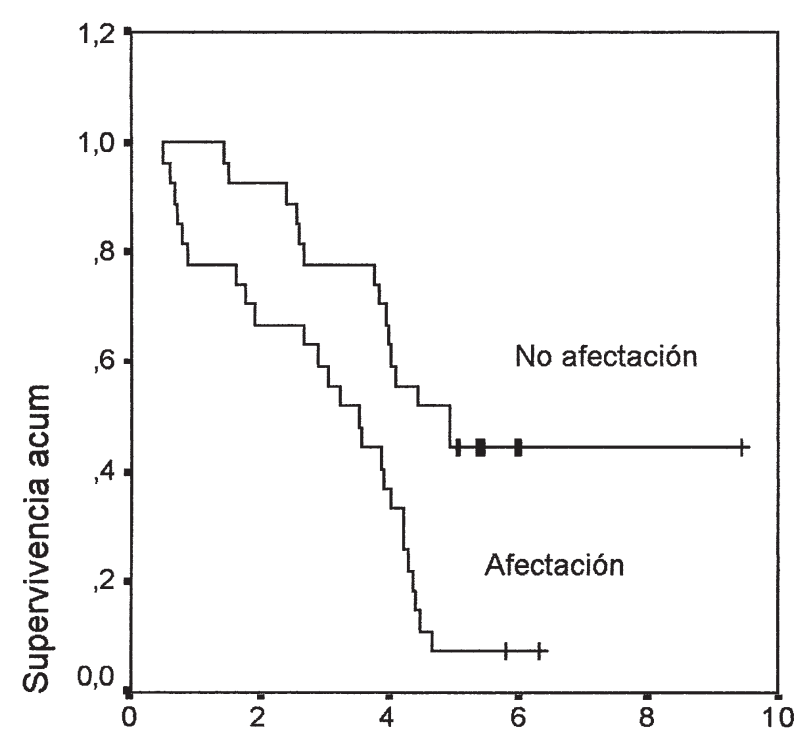

TIEMPOS

Fig. 4. Evolución de SG de los pacientes de CCR tratados con 5FU-lev según la afectació vascular linfática.

Los enfermos con afectación perineural en sus tumores, mostraron una SLE a los 5 años del $50 \%$ frente al $72.7 \%$ si no la hubo $(p=0.029)$. La SG obtenida fue del $0 \%$ cuando existía afectación perineural y del 39.9 $\%$ si no se daba esta circunstancia patológica ( $p=0.001$ ) (Fig. 5).

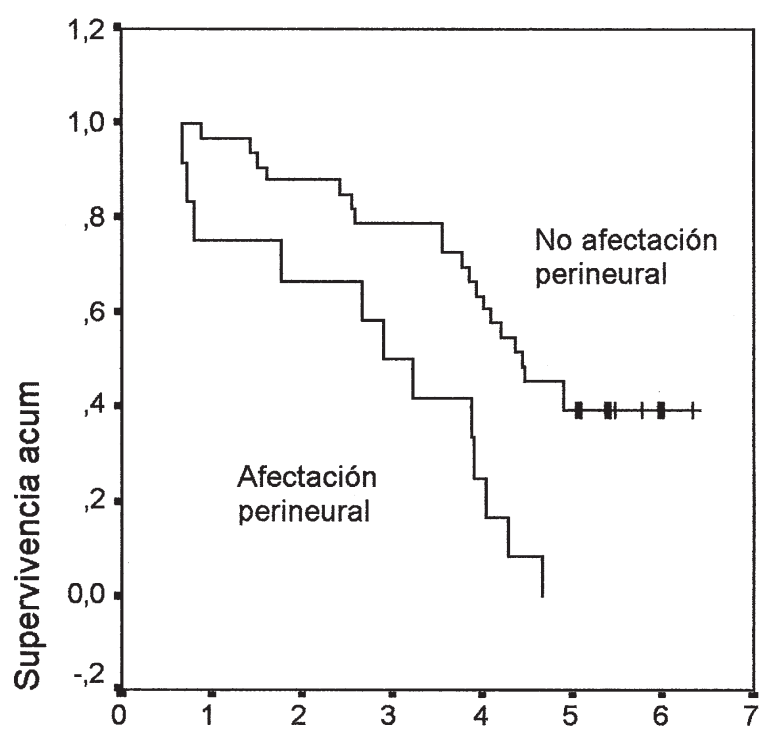

\section{TIEMPOS}

Fig. 5. Evolución de SG de los pacientes de CCR tratados con 5FU-lev segun la afectación perineural.
La supervivencia global de toda la serie ha sido de $68.3 \%$ y el porcentaje libre de enfermedad del $54 \%$, a los 5 años.

Del estudio multivariante realizado, sólo han mostrado significación pronóstica en la regresión de Cox, la afectación ganglionar con un OR $=1.3$ (IC 95\% 1.01.6) que fue estadísticamente significativo $(p=0.026)$. El resto de variables no mostraron significación estadística.

\section{Discusión}

Actualmente, el esquema adyuvante del Intergroup 0035 (5FU-Levamisol durante un año) ha sido desplazado por otros tan eficaces $y$, sobre todo, más cortos, como el esquema de la Clínica Mayo, desde que en 1996 y 1997, se comunicaron en el congreso de la American Society Clinical Oncology los resultados preliminares de los estudios de NSABP-0 $4{ }^{12}$ y el Intergroup-008913.

Entre los factores pronósticos estudiados en los pacientes operados de adenocarcinoma colorrectal estadios II y III tratados con el esquema anterior, tienen significación pronóstica, sobre todo, el número de ganglios afectos por tumor en la pieza quirúrgica, ya que la SG y la SLE, empeoran claramente según aumenta el numero de ganglios afectos por el tumor.

La importancia pronóstica del número de ganglios afectos, es conocida en la literatura desde 1932 por la clasificación de Dukes en los tumores de recto. No obstante, desde la publicación de Moertel $^{6}$ en 1990, se confirma el beneficio del tratamiento adyuvante cuando existe afectación ganglionar (N1 y N2) en los adenocarcinomas de colon que se operan con finalidad curativa.

En nuestra serie, al igual que en otros estudios ya nombrados como el del Intergroup 0035 publicado por Moertele ${ }^{6,7}$ en Annals of Internal Medicine, también se estratifica el análisis según la afectación ganglionar. En nuestro estudio agrupamos a los pacientes con NO, afectación entre ly 4 ganglios, y más de 4 adenopatías afectas, esto discrimina claramente un grupo pronóstico, tanto en el análisis Univariante como en el Multivariante puesto que en los pacientes sin afectación (NO) la SLE es del $87.3 \%$ y la SG del $87.9 \%$ a los 5 años, descendiendo a $53.7 \%$ de SLE y $58.9 \%$ de SG en pacientes con afectación tumoral de hasta 4 ganglios. Disminuyendo hasta el $45.5 \%$ tanto en SLE y SG a los 5 años cuando existe afectación de más de 4 ganglios.

En el ensayo 0035 del Intergroup ya citado, en los pacientes con afectación de 1-4 ganglios la SLE fue del 


\section{Bosch Roig y cols.}

$56 \%$ con SG del 57\%; en los de más de 4 ganglios afectos, se observó una SLE del $28 \%$ y $27 \%$ en la SG a los 5 años. Los peores resultados de este ensayo se justifican porque comunican la SLE y SG según el número de ganglios afectos, sin diferenciar los pacientes que recibieron tratamiento adyuvante y los que no.

Revisamos dos series con una población semejante a la nuestra, ya que incluyen pacientes con adenocarcinomas tanto de localización en colon como en recto, y estadios II y III tratados también con 5FU-Levamisol.

En una de ellas, un ensayo randomizado holandés de 1.029 pacientes $^{14}$, los tratados con QT adyuvante con el esquema antes descrito, logran una SLE 70.8\% y una SG a los 5 años del $78.5 \%$ en estadios sin afectación ganglionar (II) y del $51,7 \%$ y $60 \%$ respectivamente en estadio III, siendo estos resultados similares a los nuestros.

La otra serie (Reiping Tang et a ${ }^{15}$ ) incluye 538 pa- $^{-}$ cientes operados de carcinoma colorrectal en estadio III y tiene por objeto estudiar las variables pronósticas. Los autores obtienen una supervivencia a los 5 años del $69 \%$ de los pacientes con hasta 3 ganglios afectos y desciende al $49 \%-42 \%$ cuando hay 4 o más ganglios invadidos por el tumor. Estos peores resultados se explicarían porque se trata de una serie de pacientes en estadio III en la que 244 pacientes (casi el 50\%), tenían más de 4 ganglios afectos por tumor y no recibieron tratamiento adyuvante.

En nuestro estudio, hemos tratado 50 casos en estadio II (6 carcinomas de recto y 44 de colon).

Hoy día continúa la controversia de la indicación del tratamiento adyuvante de los pacientes con estadio II de colon, ya que, de los dos estudios randomizados existentes en la literatura (NSABP ${ }^{16}$ IMPACT B $2^{17}$ ), en tan sólo uno de ellos se consigue demostrar un aumento de supervivencia al tratar con quimioterapia adyuvante a este grupo de enfermos. Esta pregunta se intentará contestar con el ensayo PETACC-4 actualmente pendiente de apertura.

Otro aspecto también importante en el estadio II es el número de ganglios resecados en la pieza quirúrgica, ya que este factor va influir mucho en el número de adenopatías afectas por el tumor ${ }^{18} y$ también en la supervivencia, como lo demuestra un reciente estudio del Journal of Clinical Oncology (JCO $)^{19}$ en el que los pacientes en estadio II, tienen una supervivencia a los 5 años que pasa del $73 \%$ hasta el $87 \%$ si se logran resecar 20 ó más ganglios. Debido a esta primera razón, la actual clasificación $\mathrm{TNM}^{3}$ exige que se resequen un mínimo de 12 ganglios para considerar un tumor como N0. Nuestra casuística cumple la exigencia del TNM, ya que la media de ganglios resecados es 15.5. Esta circunstancia también justificaría, a pesar del me- nor tamaño de nuestra población, conseguir una supervivencia del $87.9 \%$ a los 5 años en pacientes en estadio II de adenocarcinoma colorrectal.

Otras variables analizadas que no han mostrado valor pronóstico en nuestra serie, pero que sí son discriminativas en otros estudios publicados son: la localización (recto o colon), el grado de diferenciación, la penetración del tumor en la pared (T) y el nivel de CEA preoperatorio, 20. Otras como la edad y los valores preoperatorios de hemoglobina y albúmina, son más controvertidas.

La falta de poder discriminatorio en nuestra serie de las primeras variables nombradas en el párrafo anterior, se justificaría por el menor tamaño de la muestra de nuestro estudio. En nuestra serie, existe un porcentaje bajo de casos con escasa diferenciación (5.5\%) respecto otras, que están en torno al $15 \%$ como en el ensayo de Moertel et $\mathrm{al}^{7}$, aunque esta variable también esta influida por la variabilidad en la interpretación por parte de los patólogos.

En nuestra serie, los tumores de recto también recibieron el tratamiento adyuvante con RT pélvica y el esquema con 5FU-levamisol, antes de la publicación del Intergroup en New England Journal of Medicine, en el que se recomienda la infusión continua de 5FU durante la radioterapia adyuvante ${ }^{21}$. Nuestros resultados en los tumores localizados recto son comparables a otras series, como la del Hospital 12 de Octubre ${ }^{22}$ o la de Tepper O'Connell 20 con 1.695 casos, tanto en porcentaje libre de recidivas, como en la supervivencia global a los 5 años. Tuvimos tan sólo 5 recidivas locales (14.28\%) en los casos de carcinoma de recto, por debajo del estudio de Cafiero et a ${ }^{23}$, en pacientes tratados con RT y el esquema de FU-Levamisol también empleado por nosotros.

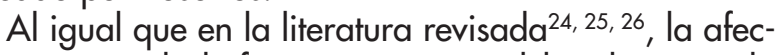
tación vásculo-linfática y perineural ha demostrado también una clara repercusión pronóstica. Creemos que este dato merece destacarse en este estudio ya que, cuando existe una de ambas variables, la SG a los cinco años desciende al $7 \%$ y $0 \%$ respectivamente (Tabla I). A pesar de todo hay que valorar estas cifras con precaución por la escasez de la muestra, ya que la información de estas variables en los tumores de nuestros pacientes fue limitada.

Entre los factores pronósticos que se estudian actualmente, y en los que se están encontrando independencia pronóstica, destaca la inestabilidad de microsatélites (variaciones en la repetición de nucleótidos, causadas por fallos del sistema de reparación del DNA durante la replicación) que se asocia a tumores de colon derecho y una mejor evolución en los tumores colorrectales $^{27-29}$. 
En la población de tumores colorrectales que nosotros hemos estudiado (estadios II y III), su valor pronóstico no es concluyente, ya que los trabajos publicados, todos, excepto dos, son series de pacientes en estadios I a IV. En un ensayo de pacientes en estadio II y III que se han tratado con quimioterapia adyuvante con $5 \mathrm{FU}$ levamisol o 5FU-Leucovorin ${ }^{30}$, la inestabilidad de microsatélites no demostró su valor pronóstico.

Habría que demostrar su valor pronóstico en grupos de pacientes en estadios de mayor riesgo de recidiva y en donde existen controversias terapéuticas (como en estadios II).

Los carcinoma colorrectales con la mutación del gen $\mathrm{K}$-ras en pacientes en estadio III, tienen peor pronostico (estudio RASCAL II) ${ }^{31}$ y además no se benefician del tratamiento adyuvante, tal y como se concluye en otro trabajo del Southwest Oncology Group ${ }^{32}$, donde también los pacientes de carcinomas colorrectales en estadio II, tenían un peor pronóstico si presentaban dicha mutación.

Asimismo, también presentan una menor supervivencia en estadios II y III en la perdida del alelo 18q, que contiene los genes supresores DCC (delected in colon cáncer gene), Smad 2 y Smad 4.

Otro factor pronóstico actual es la expresión de timidilato sintetasa en el tumor que, además de predecir la respuesta al 5FU, es por sí misma un factor pronóstico independiente en los adenocarcinomas colorrectales, ya que tienen una peor evolución en los pacientes que la expresan frente a los que no lo hacen. Si bien, dos estudios publicados en $\mathrm{JCO}$ en el $2002^{33,34}$ no consiguen demostrar una relación entre la intensidad de contenido de TS en pacientes en estadio II y III tratados con 5FU.

La aparición de nuevos conocimientos moleculares pueden tener un valor predictivo en el carcinoma colorrectal, sobre todo en estadios donde sigue habiendo controversia terapéutica (como en estadios II), o para definir el riesgo de recidiva de manera individualizada, así como su sensibilidad a los fármacos que han demostrado su actividad en carcinoma colorrectal e incluso también podrían servir como diana terapéutica.

En nuestro estudio, la SLE y SG a los 5 años es de $54 \%$ y del $68.3 \%$, estos resultados coinciden con los descritos en otra series.

En el análisis univariante, los factores pronósticos de SLE y SG a los 5 años son el numero de ganglios afectos por el tumor y la afectación vásculo-linfática y perineural intratumoral. La obstrucción intestinal tan solo resulta factor pronóstico en la SLE. Con el análisis multivariante, se mantiene como factor pronóstico el número de ganglios afectos por tumor.

\section{Bibliografía}

1. Black RJ, Bray F, Ferlay J, Parkin MD. Cancer incidence and mortality in European Union: cancer registry data and estimates for 1999. Eur J Cancer 1997; 33:1075107.

2. Gastrointestinal Tumor Study. Radiation therapy and fluorouracil with or without semustine for treatment of patients with surgical adjuvant adenocarcinoma of rectum. J Clin Oncol 1992; 10:549-57.

3. Sobin LH, CH Wittekind (eds). International Union Against Cancer: TNM Classification of Malignant Tumors, 6th Edition 2002.

4. Buyse M, Zeleniuch-Jacquotte A, Chalmers TC. Adjuvant therapy of colon cancer: Why we still don't know. JAMA $1988 ; 259: 3571-8$.

5. Laurie JA, Moertel CG, Fleming TR, et al. Surgical adjuvant therapy of large-bowel carcinoma. An evalution of levamisol and fluorouracil. J Clin Oncol 1989; 7: 144756.

6. Moertel CG, Fleming TR, Macdonal JS, et al. Levamisol and fluorouracil for adjuvant of reseted colon carcinoma. N Engl J Med 1990; 3:352-8.

7. Moertel CG, Fleming TR, Macdonal JS, et al. Levamisol and fluorouracil as effective adjuvant of reseted colon carcinoma: A final Report. Ann of Intern Med 1995; 122:321-6.

8. NIH Consensus Conference. Adjuvant for patiens with colon and rectal. JAMA 1990; 264:1444-50.

9. Boring CC, Squires TS, Tong T. Cancer Statistics. Cancer J Clin 1993; 43:7.

10. Kaplan EL, Meier P. Noparametric estimation from imcoplete observation. Journal of American Statitical Association. 1958; 53:457-81.

11. Mantel H, Haenzel W. Statistical aspects of the analisis of data from retrospective studies of disease. J Natl Cancer Inst 1959; 22:719-48.

12. Woolmark N, Rokette $H$, Mamonas EP, et al. The relative efficacy of fluorouracil + leucovorin, fluorouracil+levamisol and fluorouracil+ leucovorin+ levamisol in patients with Duckes Band carcinoma of the colon:firts report of NSABPC. Proc. Am Soc Clin Oncol 1996; 15:205/abst 460).

13. Haller DG, Catalano PJ, Macdonald JS, et al. Fluorouracil, leucovorin and levamisol adjuvant therapy for colon cancer: Five years results of INT-0089. Proc. Am Soc Clin Oncol 1997; 16:265(abst 940).

14. BG Taal, Hvan Tinteren and behalf of the NACCP group. Adjuvant 5FU plus levamisol in colinic or rectal cancer; improved survival in stage II and II. Br J Cancer 2001; 85(10): 1437-43.

15. Reiping $T$, Jeng-Yi $W$, et al. Survival impact of lynph node metastasis in TNM stage III carcinoma of the colon and rectum. JAC Surgenos 1995; 180:705-112.

16. Mamounas E, Wieand S, et al. Comparative Efficay of 


\section{Bosch Roig y cols.}

Adjuvant Chemotherapy in Patients with Dukes B Versus Colon Cancer: Results From Four National Surgical Adjuvant Breast and Bowel Projet Adjuvant Studies -NSABP- (CO1, C02, C03, and C04). J Clin Oncol 1999; 5:1349-55.

17. Eficcacy of adjuvant Fluorouracil and folinic acid in B2 colon cancer IMPACT B2. J Clin Oncol 1999; 17:13561363

18. Wong J, Severino R, Barbera M, Paul T, Namiki T. Number of Nodes Examined and Staging Accury in Colorectal Carcinoma. J Clin Oncol 1999; 17:2896-900.

19. Le Voyer TE, Sigurdson ER, Hanlon AL, Mayer RJ, Macdonald JS, Catalano PJ, Haller DG. Colon Cancer Survival Is Associated With Increasing Number of Lymph Nodes Analyzed: A Secondary Survey of Intergroup Trial INT-0089. J Clin Oncol 2003; 21:2912-9.

20. JE Tepper $\mathrm{O}^{\prime}$ Connell et al. Adjuvant therapy in rectal cancer: analysis of stage, sex and local control-Final Report of Intergroup 0114. J Clin Oncol 2002; 20:174450.

21. O'Oconell M, Martenson JA, et al. Improving adjuvant therapy for rectal cancer by combining protracted-Infusion fluorouracil with radiation therapy after curative surgery. N England J Med 1994; 331:502-7.

22. Caballero $P$, Pérez MA, Cruz J, et al. Carcinoma de alto riesgo: factores pronósticos y resultados del tratamiento combinado postquirúrgico en el Hospital Universitario Doce de Octubre. Oncológica 2000; 23:486-94.

23. Cafiero $\mathrm{F}$ et al. Preliminary analysis of randomized clinical trial of adjuvant postoperative RT vs postoperative RT plus 5FU and Levamisol in patients with TNM stage II-III resectable rectal cancer. J Sur Oncol 2000 . 75:80-8.

24. Shirounzu K et al. Prognostic Evaluation of Perineural Invasion in Rectal Cancer. Am J Surgical 1993; 165; 2337.
25. Bognel C et al. Prognostic Value of Neural Invasión in Rectal Carcinoma: Multivariate Análisis on 399 Patients UIT Curative Resection. Eur J Cancer 1995; 31 ;894-8.

26. Compton C, Fenoglio-Preiser CM, Petigrew N, Fielding P. American Joint Comite of Cancer. Prognosis factor consensus conference. Colorectal Working Group. Cancer 2000; 88:1739-57.

27. Halling K, French A, Shannon K, et al. Microsatellite inestabily and 8p allelic imbalance in stage B2 and C colorectal cancers. J Natl Cancer Inst 1999; 91:1295303.

28. Tribbodeau S, Bren G, Schaid D. Microsatellite inestabily in cancer of the proximal colon. Science 1993; 260: 816-9.

29. Gryfe R, Kim H, Hsieh, et al. Tumor microsatellite instability and clinical outcome in young patients with colorectal cancer. N Engl J Med 2000; 342:69-77.

30. Ribic CM, Sargent DJ, Moore MJ, et al. Tumour microsatellite inestability (MSI) and benefit of 5FU based chemotherapy in stage II, III colon cancer: a pooled molecular reanalysis of randomised chemotherapy trials. Proc Am Soc Clin Oncol 2002; (abst 509).

31. Andreyev HJ, Norman AR, Cunninghan D, et al. Kristen ras mutation in patients with colorectal cancer: the RASCAL II study. Br J Cancer 2001; 85:692-6.

32. Ahnen D, Feigl P, Quan G, et al. Kras mutacion and P53 overexpresion predict the clinical behavior of colorectal cancer: a Soutwest Oncology Group. Cancer Res 1998; 58:1149-58.

33. Llegra CJ, Parr AL, Wold LE, et al. Investigation of the prognostic and predictive value thymidylate synthetase in colorectal cancer. J Clin Oncol 2002; 20:1735-43.

34. Edler D, Glimelius B, Hallstron M, et al. Thymydilate synthase expresion in colorectal cancer: a pronostic and marker of benefit from adyuvant fluorouracil-based chemotherapy. J Clin Oncol 2002; 20:1721-8.
Correspondencia:

Dr. C. Bosch Roig

Unidad de Oncología Médica

Hospital de Día

Hospital Universitario Dr. Peset

Av. Gaspar Aguilar, 90

E-46017 Valencia

E-mail: bosch_car@gva.es oncologia_peset@gra.es 